\title{
Efficiency of PRI and WRR DiffServ Scheduling Mechanisms for Real-Time Services on UMTS Environment
}

\author{
Nuno Vasco Lopes, Maria João Nicolau and Alexandre Santos \\ nunovasco@gmail.com, joao@dsi.uminho.pt, alex@di.uminho.pt \\ Engineering School, University of Minho \\ 4710 Braga, Portugal
}

\begin{abstract}
The next generation of mobile phones will be probably all-IP based enabling users to access Internet services. In order to make this possible a satisfactory quality of service, at least equal to the fixed Internet, must be ensured. To achieve this goal an End-to-End QoS system must be constructed. Another fact is the dominance of IP over other technologies due, in large measure, to its characteristic of working with heterogeneous technologies. Consequently, being IP the common denominator on a heterogeneous environment, it is important to develop Endto-End IP QoS guarantees for the different applications over distinct access technologies. This is particularly important for cellular wireless networks due to the ever growing expansion of mobile phone users. One way to contribute to this goal is to apply DiffServ QoS mechanisms to UMTS technology in order to model an End-to-End QoS communication system. A mapping of DiffServ CodePoints into UMTS classes can be applied in order to get efficient PHB configurations. This paper proposes an architecture to support end-to-end quality of service to several application services running on mobile UMTS user agents and communicating with servers located in a wired internet. The proposed architecture is based on a DiffServ model, where QoS parameters are set either by the user agent or by the SGSN. In particular, RED queue management and PRI or WRR scheduling policies are enforced. Different UMTS traffic classes are mapped into different DiffServ parameters. The performance of this architecture has been evaluated by simulation using NS, assuming different network load scenarios. In particular, the delay and packet loss experienced by VoIP, Video, FTP and HTTP traffic are evaluated in the cases of PRI and WRR scheduling policies, and compared to those measured when DiffServ is not implemented. Finally, a revenue function to estimate the profits that an ISP could expect by using a DiffServ implementation on IP UMTS core routers is proposed.
\end{abstract}

Index Terms-End-to-End QoS, UMTS, DiffServ, Scheduling, WRR, PRI, QoS, Mapping, QoS Metrics, Pricing.

\section{INTRODUCTION}

At present, efforts are being made to provide end-to-end Quality of Service (E2E QoS) in both $3 \mathrm{G}$ mobile radio systems (3GPP/3GPP2) and IP infrastructure (IETF) towards the convergence of both standards. The market is imposing new challenges specially for real-time and quality-assured services in mobile communications world which tends to be all-IP based [1]. Therefore, to achieve a seamless convergence of fixed and mobile services and voice/video and also elastic applications coexistence a common IP-based platform is very

Nuno Lopes is supported by an FCT Grant (SFRH/BD/35245/2007) important to endow the Internet with fine QoS resolution. It can be expected that QoS parameters for wireless users are more complex than those for wired packet access[2]. On the other hand the increasing bit rates supported in $3 \mathrm{G}$ radios together with the increasing processing power of mobile terminals provide opportunities for introducing various realtime and interactive multimedia applications and services to mobile users. However intrinsically, IP does not offer guarantee of service and providing IP with QoS is a difficult task. The unpredictability nature of wireless networks with high loss and traffic variability turns this task even more difficult. Hence, to improve the Internet architecture for different UMTS classes it is necessary that routers perform distinct treatment for each type of traffic. So, the existent QoS mechanisms, and eventually new ones, need to be exhaustively tested on heterogeneous environments to see how well they perform. DiffServ mechanisms were chosen because they were designed to be a simple QoS architecture that can provide E2E services across different operator's domain without requiring a complex signalling process for every connection. To achieve this DiffServ uses key concepts like aggregate flows and Per-HopBehaviours (PHBs).

In this paper a study is presented where DiffServ mechanisms, usually used in fixed IP networks, were applied to UMTS mobile networks. Therefore the focus will be on analysis of E2E QoS provisioning on a mixed network environment composed by an IP external domain and by an UMTS domain.

This paper is organized as follows: after a brief review of State-of-the-Art and concepts to accommodated QoS in mobile networks, a description of the methodology used is presented. Simulation results with our network model architecture and a volume-based pricing revenue function, based on a general fixed pricing structure is also proposed. Finally, section 7 presents the conclusions of the work.

\section{Previous Work}

In [3], Abd-Elhamid M. Taha et al. propose some mobile IP QoS extensions to improve mobility between domains. The MIP handover between domains, still suffers from long delays and the reassessment of gateway protocols is proposed in order to facilitate the seamless mobility. Abd-Elhamid M. Taha et al. recommend the MPLS-based MIP solution, instead of RSVP 
solution, because the first solution provides a superior level of abstraction and better scalability. In [4], Jukka Manner et al. discuss the problems related to QoS provision to mobile terminals and identify the existing solutions and the future work needed. S. Baudet et al. [5] propose DiffServ mechanism implementation at the IP level, and MPLS functions at the level two, but lacks of results demonstration. Haibo Wang et al. investigated an end-to-end QoS model on UMTS environment [6]. Based on optimizing the mapping of UMTS classes, access control, buffering and scheduling, they developed an algorithm to improve the performance in terms of delay and loss while increasing the bandwidth usage. However this article does not explain clearly the interactions between different mechanisms used to achieve end-to-end QoS. Hemant Chaskar et al., in [7], describe how to use the DiffServ and MPLS to implement QoS in UMTS. The PDP (Packet Data Protocol) signaling is used to implement the various QoS contexts on border nodes (SGSN and GGSN). In [8], IP mobile network issues and its interactions with DiffServ architecture for UMTS traffic are discussed, also analysing a core IP network which is able to support 3GPP QoS requirements with tight service-level requirements. Despite the quality of existing related work some topics still require more in depth coverage, as follows:

- Many E2E QoS frameworks were proposed but solid implementation and simulation outputs are still missing;

- QoS attributes mapping are designed but not all implemented;

- None of these frameworks give a clear picture about the impact of mixed traffic;

- None of these studies discuss QoS aspects for real-time and non real-time traffic.

\section{RELATED CONCEPTS}

A UMTS network can be viewed as a special IP network, which offers IP connectivity to IP mobile terminals. To provide such a mobile connectivity service, UMTS must implement additional functionalities. By using shared radio resources, mobile users can access remote Packet Data Networks (PDN) by means of a remote access router, called GGSN, that provides virtual connection to remote PDN. The GGSN also controls the end-to-end IP QoS bearer service towards the remote terminal.

There are many different end-to-end issues for the connection of a UE (user equipament) to a UTMS network. Figure 1 shows the way end-to-end QoS may be accomplished for a significant number of scenarios [9]. Scenario 1 of TS 23.207 [10] has been chosen, where the GGSN supports DiffServ edge functions and the IP backbone network is DiffServ enabled.

The application layer (e.g. SIP/SDP) identifies the QoS requirements. These QoS requirements (e.g. TS 23.228 [11] describes interworking from SIP/SDP to QoS requirements) are mapped into PDP context parameters in the UE. In this scenario, the control of the QoS over the UMTS access network (from the UE to the GGSN) may be performed either from the terminal (using the PDP context signaling), or from the SGSN (by data subscription). The GGSN will apply receiver control DiffServ edge functions and may reclassify

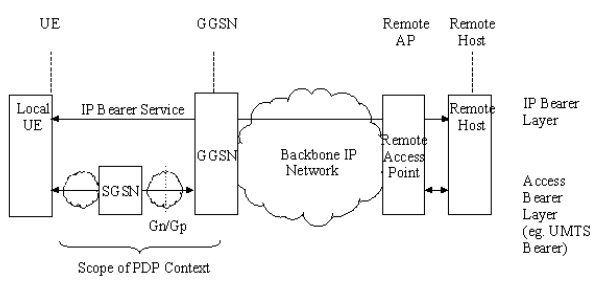

Figure 1: Network Architecture for QoS Conceptual Models [3GPP TS 23.207 V7.0.0 (2007-06)]

data (remarking the DiffServ Code Point (DSCP)). Figure 2 shows the way end-to-end QoS is provided in this scenario: a local mechanism in the UE uses the PDP context for QoS over the UMTS access network and the IP backbone network uses DiffServ to provide QoS guarantees. The GGSN provides the inter-working between the PDP context and the DiffServ functions.

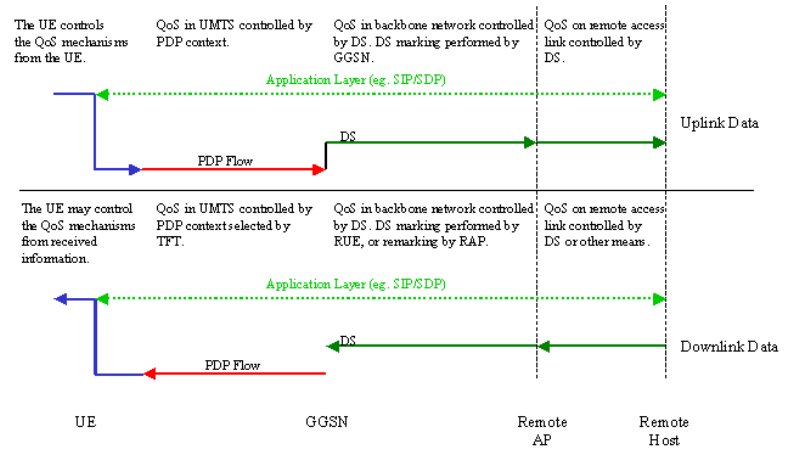

Figure 2: Local UE does not provide IP DiffServ capabilities [3GPP TS 23.207 V7.0.0 (2007-06)]

\section{A. UMTS QoS Classes}

When defining the UMTS QoS classes, also referred to as traffic classes, the air interface restrictions and its limitations have to be taken into account. It is not reasonable to define complex mechanisms, as has been the case in fixed networks, due to different characteristics of the air interface. The QoS mechanisms provided in the cellular network have to be robust and capable of providing reasonable QoS resolution. There are four different QoS classes: Conversational class; Streaming class; Interactive class; Background class. The main distinguishing factor between these QoS classes is how delay sensitive the traffic is: Conversational class is meant for traffic which is highly delay-sensitive, while Background class is the least delay-sensitive one. Conversational and Streaming classes are mainly intended for real-time traffic flows, the main distinction being on how delay-sensitive the traffic is. Conversational real-time services, such as video telephony, are the most delay-sensitive applications and those data streams should be carried in the Conversational class. Interactive and Background classes are mainly meant to be used for traditional Internet applications such as WWW, Email, Telnet, FTP and News. When compared to Conversational and Streaming classes, they provide better error rate by means of channel 
coding and retransmission. Traffic in the Interactive class has higher priority in scheduling than Background class traffic, so background applications use transmission resources only when interactive applications do not need them. This is very important in wireless environment where the bandwidth is low compared to fixed networks.

1) Conversational class: For real time conversation the fundamental QoS characteristics are: preserve time relation (variation) between information entities of the stream; conversational pattern (stringent and low delay).

2) Streaming class: For real time streams the fundamental QoS characteristics are: preserve time relation (variation) between information entities of the stream.

3) Interactive class: For interactive traffic the fundamental QoS characteristics are: request response pattern; preserve payload content.

4) Background class: For background traffic the fundamental QoS characteristics are: the destination is not expecting the data within a certain time; preserve payload content.

\section{QoS ToolSeT}

This section describes the combination of tools used to achieve the overall design objectives of our network model. These fall into the following categories: classification and marking; policing and shaping; congestion avoidance and congestion-management. At the edge router packets will be marked or re-marked, and all classification is to be processed based on markings. At this point, packets might be discarded by a policer (e.g. if they exceed the committed information rate) or by the congestion-avoidance mechanism (e.g. if the packet exceeds a given threshold). Those packets which are not discarded are subject to scheduling in order to prioritize packets that belong to higher priority UMTS classes.

\section{A. Scheduling and Queuing}

When an interface experiences congestion the process of deciding which packet to transmit is an important issue. Thus, the key point is to give a different treatment to packets that belong to a class with tight QoS requirements. Consequently, the decision on the type of scheduling to be used is based on its capacity to adapt to the QoS Class requirements. In order to decide on this we have examined the Strict Priority and the Weighted Round Robin scheduling mechanisms.

\section{Methodology}

The main objective of end-to-end simulations is to capture all the aspects of the system design and examine the impact that IP with QoS support has on UMTS environment. The case study is a simulation scenario, which consists in hybrid traffic transmission from a fixed IP network to UMTS mobile terminals, in the heart of a business city. The simulation platform used to implement the network model is the Network Simulator [12], version 2.29. The model has sixteen traffic servers - seven VoIP, seven Video, one HTTP and one FTP located in the fixed network, and each of them transmits its data to UMTS mobile terminals (see Figure 3). By means of this model we intend to study DiffServ mechanism behavior when the network is overloaded. The network bottleneck is located in the GGSN - SGSN link. In order to cause more or less congestion on this link, four bandwidth settings were used: BW1 - 2Mbps; BW2 - 0.8Mbps; BW3 - 0.6Mbps; and BW4 - 0.4Mbps (see Table I). Each of these bandwidth settings will be named as No Congestion (BW1), Light Congestion (BW2), Heavy Congestion (BW3) and Very Heavy Congestion (BW4), respectively. In order to compare results we simulated the same network model, with and without DiffServ, for the four configurations mentioned. On DiffServ simulations two scheduling mechanisms have been used: PRI (Priority) and WRR (Weighted Round Robin) (see Table II).

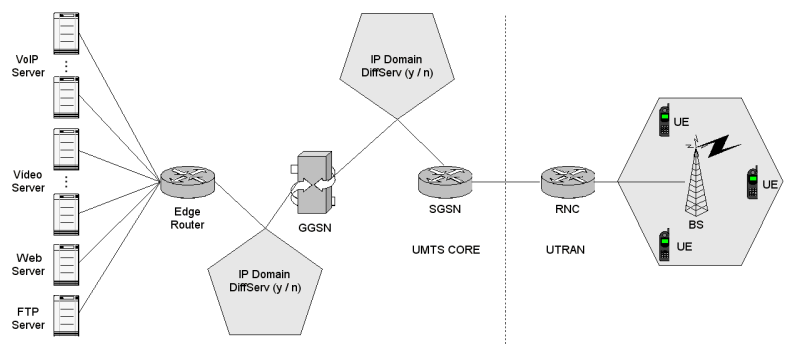

Figure 3: Simulation Topology

This simulation environment will enable the validation of DiffServ mechanisms, as a possible solution in order to provide end-to-end QoS over heterogeneous networks. To achieve this objective, three main development efforts were conducted: mapping DiffServ DSCP into UMTS classes; queues management; and scheduling optimization.

The simulation model uses four application traffic types: voice, video, interactive and background. The congestion link is set to be between GGSN and SGSN. The radio transmission channels has been configured as dedicated channels (DCH), in order to avoid the dependency among them, with $384 \mathrm{kps}$ of capacity.

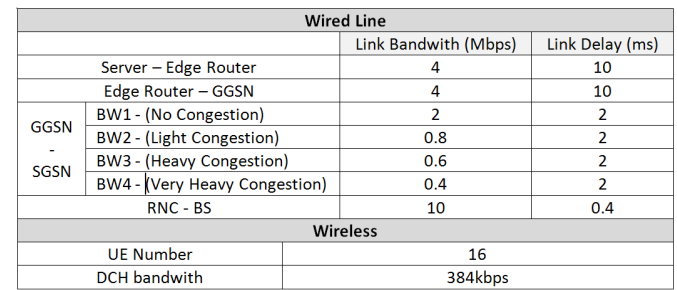

Table I: NS simulation parameters

In what concerns the queue management, DropTail method has been used in all links, with a single exception on the link between GGSN and SGSN (where RED (Random Early Detection) has been used) (see Table III). This mechanism uses the average of queue occupation as a parameter. If traffic is below a minimum value all packets pass through; If not, the probability to discard the packets grows linearly until a maximum threshold; Once attained the maximum threshold all the packets are discarded. The DropTail policy is a very simple algorithm that puts packets on the queue by the same 
order of their arrival; If the queue limit is reached, all the arrived packets are discarded.

\begin{tabular}{|l|c|c|c|c|}
\hline \multicolumn{1}{|c|}{ Application } & VoIP & Video & HTTP & FTP \\
\hline PRI (kbps) & 800 & 300 & 150 & 50 \\
\hline WRR (weight) & 10 & 7 & 2 & 1 \\
\hline
\end{tabular}

Table II: Schedulings parameters

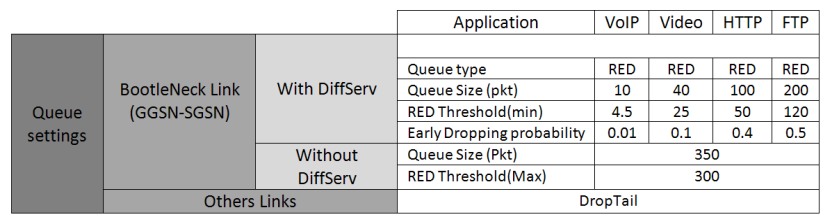

Table III: Links characteristics

\section{A. Traffic Models}

An important point in the analysis and design of networks, is the development of a rigorous analytical model for each traffic type. This section describes the four classes traffic models that have been used.

1) Voice: A typical telephone conversation is marked by active periods $(\mathrm{ON})$ of conversation and by silence periods (OFF) when users are listening, so it can be modelize by the two Markov process as suggested by ITU-T [13]. The periods ON and OFF will be modeled by an exponential distribution with an average duration of $350 \mathrm{~ms}$ when $\mathrm{ON}$ and an average duration of $650 \mathrm{~ms}$ when OFF. The duration of each state follows an exponential distribution. During the activity periods it generates in fixed intervals, packets with 372 bytes (fixed size). To code the voice traffic the AMR standard was utilized with a throughput of $12.2 \mathrm{Kbps}$. It was assumed that 10 frames per packet are transmitted. Each AMR frame may vary between 32 and 35 bytes, depending on the options used (octet-alignment, CRC and interleaving) and on AMR RTP payload header [14]. This leads to packet sizes of 372 bytes: 10 frames per packet: 20 (IPv4) + 8 (UDP) + 12 (RTP) + 332 (max RTP payload for 10 AMR frames).

2) Video: There are several video traffic models, even for codecs enabling highly variables bit rates, but in this paper an advanced conventional CBR server has been chosen to model video traffic. The reasons for this choice were: most of commercial videos are CBR, CBR has a high predictability and is a good compromise to analyse quality fluctuations on account of losses or received bitstream rate variations. The video coding, was configurated with a throughput of $47.7 \mathrm{Kbps}$ and with packets of 500 bytes (RTP payload) [14], leading to packet sizes of 540 bytes: $20(\mathrm{IPv} 4)+8$ (UDP) +12 (RTP) + 500 (max AMR RTP payload).

3) Web Browsing: For HTTP model characterization the sessions have been split into ON/OFF periods representing respectively, web-pages downloads and intermediate times of reading. In addition, as web traffic is self-similar, the sessions were generated upon ON/OFF periods [15], [13]. Taking into account mobile device limitations and the user/device interactions, HTTP applications have been mapped into three levels of abstraction: sessions, pages and objects. One session could contain one or more pages and each page could contain one or more objects. Based on previous results (as [16]) showing large packet sizes used in Internet, a packet size of 500 bytes has been chosen.

HTML pages are generated by the random model HTMLpagepool implemented in the NS2 plataform [12]. The HTML page size is computed as being the main object size plus the number of objects embedded in that page. The traffic generation parameters used are summarised in Table IV.

\begin{tabular}{|c|c|c||c|}
\hline Traffic parameters & Distribution & HTTP - Average value & FTP - Average value \\
\hline \hline Session size & Exp & 5 & 1 \\
\hline Session inter-arrival time & Exp & 7 & 720 \\
\hline Page size & Exp & 3 & 1 \\
\hline Page read time & Exp & 0.1 & 0 \\
\hline Object size & Exp & $20 \mathrm{~KB}$ & $5 \mathrm{MB}$ \\
\hline
\end{tabular}

Table IV: HTTP and FTP parametrization values

4) FTP: In the FTP application a session is model as a sequence of file transfers followed by reading periods. Therefore, FTP sessions behave similar to HTTP requests, but without the page abstraction level. The traffic generation parameters used are shown in Table IV.

\section{B. Traffic Distribution}

After modelling traffic sources, next step is to choose the amount of data that each source should generate. The WiMax Forum study [17] was used to shape this distribution and the traffic distribution foreseen by WiMax Forum to year 2020 was also used. Table $\mathrm{V}$ summarizes the characteristics of the adopted models and the traffic distribution for each type of traffic.

\begin{tabular}{ccccc}
\hline & VolP & Video Streaming & Web Browsing (HTTP) & FTP \\
\hline Application Protocol & Exponential & CBR & HTTP & FP \\
\hline Transport Protocol & UDP & UDP & TCP & TCP \\
\hline Packet Size(bytes) & 372 & 540 & 500 & 500 \\
\hline Rate(kbps) & $12,2(\times 7$ sources $)=85$ & $47,7(\times 7$ sources $)=333$ & 160 & 484 \\
Rate(\%) & 8 & 31 & 15 & 46 \\
\hline Interleaving(ms) & 20 & 2 & 7 & 20 \\
\hline
\end{tabular}

Table V: Traffic characteristics

The models described above take into account the inherent characteristics of each traffic class, as well as the four classes of UMTS service organization.

\section{Simulation Analysis}

In order to show the benefits of DiffServ mechanisms the same network model has been analyzed in congestion and no congestion states. In the congested case three levels of saturation have been used (Light, Heavy, Very Heavy). All the congested scenarios were analysed either in the presence or in the absence of DiffServ mechanisms. NS simulations have been conducted to evaluate the efficiency of two scheduling disciplines - WRR and PRI - in the context of UMTS. The traffic used as input in the simulation is based on a real 
world study of traffic breakup across various classes. The average jitter and delay of VoIP packets is computed and compared to the recommendation of the ITU. Results are put into perspective with link utilization and profitability, based on a revenue model. The overload stressing conditions that have been analyzed are: Light:+13\%; Heavy;+43\%; Very Heavy: $+54 \%$.

All simulated scenarios assume that overload is imposed by all traffic sources and that there are always enough resources to accommodate all real-time traffic generated (real-time traffic alone will not lead to congestion).

\section{A. UMTS QoS Analysis using PRI and WRR}

1) VoIP analysis: Figure 4 illustrates the voice traffic average delay in the three congestion scenarios. It is visible that delay are significantly reduced in all the congested scenarios when DiffServ mechanisms are implemented. It can be noticed also that PRI scheduling presents smaller values for both delay and jitter than those attained with WRR. When DiffServ is used, either with PRI or WRR, QoS requirements for VoIP service are always kept within the limits imposed by ITU recommendations in the three congested scenarios.

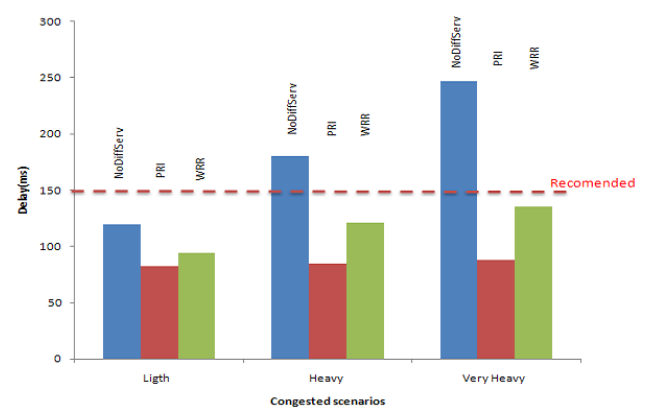

Figure 4: VoIP average Delay in all congestion scenarios

Figures 5 and 6 show the instant jitter for each VoIP traffic source in the heavy congestion scenario. It can be seen that PRI scheduling mechanism reduce the maximum jitter on the order of magnitude of 10 (notice that jitter yy scales on Figures 5 and 6 are different).

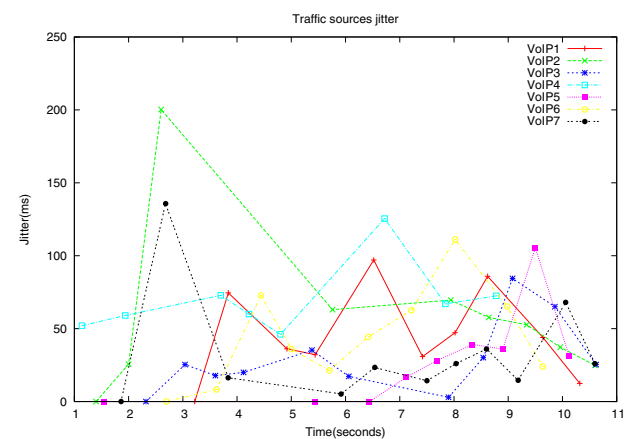

Figure 5: VoIP flows in heavy congestion scenario without DiffServ

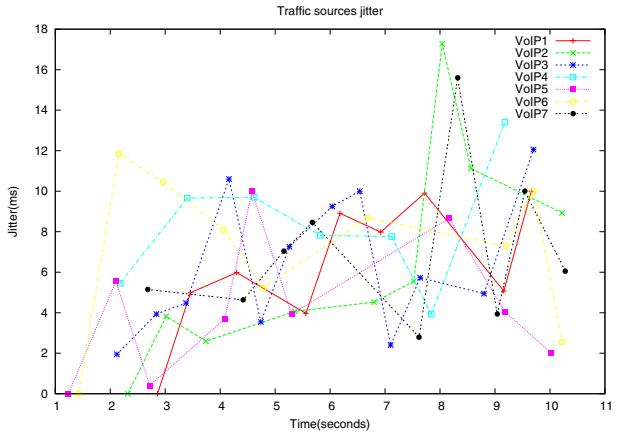

Figure 6: VoIP flows in heavy congestion scenario with PRI scheduling mechanism

2) Video analysis: In what concerns to the video traffic, the light scenario (overload 13\%) evidences that when DiffServ mechanisms are applied all the three QoS parameters delay, jitter and lost are better fulfilled and satisfy ITU recommendations, whichever scheduling is used. For the heavy congestion scenario only WRR scheduling mechanism allows coping with ITU recommendations; when PRI is used (same overload) the average delay rises to a value of $361 \mathrm{~ms}$, meaning that no video service is within the recommended $250 \mathrm{~ms}$ delay. In the very heavy congestion scenario none of scheduling mechanisms, either PRI or WRR is able to fulfill the QoS requirements (see Table VI).

When comparing scheduling mechanisms one can conclude that WRR is able to keep within the ITU recommendations for an overload of $43 \%$, whereas the PRI only meets the ITU recommendations for an overload of $13 \%$.

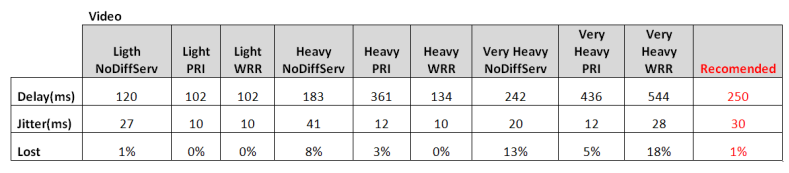

Table VI: Video QoS results

\section{B. QoS and Revenue Analysis.}

Results show that if one applies DiffServ mechanisms, even in scenarios with different levels of congestion, the priority classes with real time services show a significant increase of efficiency both in QoS and in the data volume transmitted, as shown in Table VII.

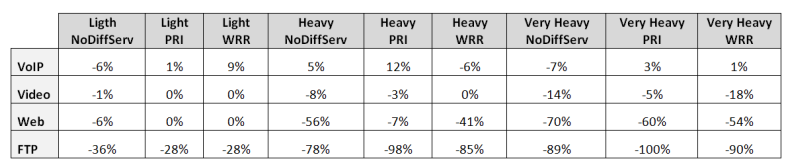

Table VII: Data transmission percentage with network congested

Apart from the fact that VoIP traffic is kept within strict QoS limits, data transmission efficiency also increases, leading to achieve an higher utilization percentage of the available bandwidth (see Table VIIIa). 


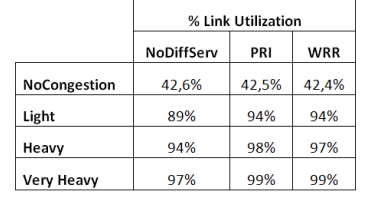

(a) Link utilization, percentage of Bandwidth

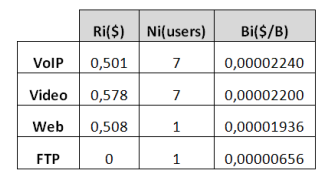

(b) Assumed prices \& number of users by priority of service
Table VIII: Link utilization and Discrimination prices

Using the same experimental setup it has been possible to analyze the importance of DiffServ mechanisms, when applied to the provision of real-time services in UMTS environments, in two separate contexts: the purely technical context and the economic contex in terms of potentiating the increase in commercial revenue.

In order to show the DiffServ implementation advantages to ISPs, a revenue function has been applied to each of the four scenarios studied. In this revenue function, each priority class has a fixed payment component and a variable one. Prices depend on amount of transferred data and its priority. The revenue function is computed as $N_{i} \times R_{i}+V_{i} \times B_{i}$ where: $N_{i}$ : number of users of priority $i ; R_{i}$ : fixed payment of users of priority $i ; V_{i}$ : transferred data volume of priority $i$; and $B_{i}$ : per byte charge of priority $i$.

Based on the work published in [18] a two-tariff price is presumed. The function has different discrimination prices and fixed charges for each UMTS class, with the values shown in the Table VIIIb. In this model, the network operator charges the user with a fixed charge and a per volume charge, being per byte charge and the fixed charge in accordance with service class. It means that the user charge is determined by the consumed resource, the average user demand, and the level of service guaranteed to the user [19].

After applying the revenue function to the experimental setups, one may conclude that if the ISP implements the DiffServ mechanisms on the UMTS network core profits can increase, in all the three congestion scenarios. Table IX illustrates the revenue function results as computed for all the scenarios and its comparison with the no congestion scenario (gain decrease, in percentage).

\begin{tabular}{|c|c|c|c|c|c|c|c|c|c|}
\hline $\begin{array}{c}\text { No } \\
\text { Congestion }\end{array}$ & $\begin{array}{c}\text { Ligth } \\
\text { NoDiffServ }\end{array}$ & $\begin{array}{c}\text { Light } \\
\text { PRI }\end{array}$ & $\begin{array}{c}\text { Light } \\
\text { WRR }\end{array}$ & $\begin{array}{c}\text { Heavy } \\
\text { NoDiffserv }\end{array}$ & $\begin{array}{c}\text { Heavy } \\
\text { PRI }\end{array}$ & $\begin{array}{c}\text { Heavy } \\
\text { WRR }\end{array}$ & $\begin{array}{c}\text { Very Heavy } \\
\text { NoDiffServ }\end{array}$ & $\begin{array}{c}\text { Very } \\
\text { Heavy PRI }\end{array}$ & $\begin{array}{c}\text { Very Heavy } \\
\text { WRR }\end{array}$ \\
\hline$\$ 25,88$ & $\$ 24,34$ & $\$ 24,95$ & $\$ 24,97$ & $\$ 20,45$ & $\$ 22,31$ & $\$ 21,39$ & $\$ 18,80$ & $\$ 19,81$ & $\$ 19,03$ \\
\hline $0 \%$ & $-6 \%$ & $-4 \%$ & $-3 \%$ & $-21 \%$ & $-14 \%$ & $-17 \%$ & $-27 \%$ & $-23 \%$ & $-26 \%$ \\
\hline
\end{tabular}

Table IX: Revenue function results

\section{CONCLUSIONS}

In this paper we have analyzed and pointed out the advantages of applying DiffServ mechanisms in UMTS network core when the network is facing stressing traffic conditions, leading to congestion.

It has been shown that even in congested scenarios the ITU QoS recommendations are met up to an overload of $13 \%$ whichever scheduling mechanisms are used. It has also been noticed that when WRR is used the system is able to meet the ITU QoS requirements for real-time services, even for an traffic overload of $43 \%$.

Also, simulation results have shown that there is an efficiency increase in data transmission for priority classes that are associated to real time services like VoIP and Video, and simultaneously the ITU QoS recommendations for real time traffic are preserved. Furthermore, simulations results show a substantial enhancement for QoS parameters delay, jitter and lost.

Thirdly, we have also shown that the DiffServ mechanism led to an increase of the link usage, enabling a more effective management of the available bandwidth.

Finally, to get evidence of DiffServ implementation gains, a revenue function has been applied in order to estimate the profits that ISP would expect. The revenue function, with a fixed and a per byte charge, is able to cope both with traffic classes and traffic volume. It is evident that, apart from quality assurance, the revenues would increase if DiffServ would be deployed, even in all of the congested scenarios.

\section{REFERENCES}

[1] 3GPP, "Overview of 3gpp release 5," ETSI Mobile Competence Centre, Tech. Rep., September 2003.

[2] S. Dixit and R. Prasad, Wireless IP and Building the Mobile Internet, A. House, Ed. WPMC'01, 2003.

[3] A.-E. M. Taha and H. S. Hassanein, "Extensions for internet qos to mobile ip: a survey." IEEE Communications Magazine, pp. 132-139, May 2005.

[4] J. Manner, A. Lopez, A. Mihailovic, H. Velayos, E. Hepworth, and Y. Khouaja, "Evaluation of mobility and qos interaction computer networks," Elsevier Science Publisher, vol. 38, pp. 137-163, February 2002.

[5] S. Baudet and et al., "Qos implementation in umts networks," Alcatel Telecommunications Review, vol. 1st Quarter, 2001.

[6] H. Wang, D. Prasad, O. Teyeb, and H. P. Schwefel, "Performance enhancements of umts networks using end-to-end qos provisioning," WPMC, 2005

[7] H. Chaskar and R. Koodli, "Mpls and diffserv for umts qos in gprs core network architecture," INET, 2001.

[8] Cisco, "Ip class of service for mobile networks," Cisco Press, 2005.

[9] T. Szigeti and C. Hattingh, End-to-End QoS Network Design: Quality of Service in LANs, WANs, VPNs, I. Cisco Systems, Ed. Cisco Press, 2005.

[10] 3GPP, "End-to-end quality of service (qos) concept and architecture (release 7)," 3GPP, Technical Specification 23.207, July 2007.

[11] ETSI, "Ip multimedia subsystem (ims); stage 2," ETSI, Technical Specification 123 228, January 2008.

[12] K. Fall and K. Varadhan, The ns Manual, UC Berkeley, LBL, USC/ISI, and Xerox PARC., October 2006. [Online]. Available: http://www.isi.edu/nsnam/ns/ns-documentation

[13] ITU-T, "Telephone transmission quality objective measuring apparatus: Artificial conversational speech," ITU-T, Recommendation P.59, March 1993.

[14] 3GPP, "Transparent end-to-end packet switched streaming service (pss); rtp usage model," 3GPP, TR 26.937 Version 6.0.0, March 2004.

[15] J. Cao, W. Cleveland, Y. Gao, K. Jeffay, F. Smith, and M. Weigle, "Stochastic models for generating synthetic http source traffic," IEEE INFOCOM, 2004.

[16] K. C. Claffy and S. McCreary, "Internet measurement and data analysis: passive and active measurement," ASA stat, 1999.

[17] W. Forum, "A review of spectrum requirements for mobile wimax equipment to support wireless personal broadband services," WiMAX Forum, Tech. Rep., September 2007. [Online]. Available: http://www.wimaxforum.org/

[18] H.-C. Jang and B. Lu, "Pricing-enabled qos for umts/wlan network," JCIS, October 2006.

[19] X. Wang and H. Schulzrinne, "Performance study of congestion price based adaptive service," in ICC (3), 2000, pp. 1665-1669. 